showing definite promise for short-rotation arable lye, such as occurs in the South Island. Interesting work has been carried out by tho Plant Chemistry Iaboratory on plant growth-substances and their effect in promoting the rooting of cuttings, and particularly effective results have been obtrined with $\beta$-indolylbutyric acid.
The report also refers to the work of the Leather and Shoe Research Association, the Wool Mranu. facturers Research Association, which has continued the study of the raw wool scouring process, to radio research, the activities of the Social Science Research Bureau and of the Dominion Iaboriztory and Geological Survey.

\title{
THE CRAB NEBULA: A PROBABLE SUPER-NOVA
}

$\mathrm{T}$ HE Crab Nebula in Taurus, which has the distinction of being No. 1 in Messier's Catalogue, is of peculiar interest, as is shown by articles published respectively in $L$ 'Astronomie (August 1939) and in the Telescope (September 1939). Theso articles summarize the results of several technical papers published on this subject during the past few years. It seems likely that the Crab Nebula was first recorded by John Bovis, an English physician, in 1731. It was rediscovered in 1758 by the French astronomer, Charles Messier, and was later tho subject of careful scrutiny by Sir IVilliam Herschel. It was observed with the great reflector at Parsonstown, Ireland, by Lord Rosse, whose drawing of the nebula, published in 1844, probably suggested its namo. It remained, however, for astronomical photography to show, first in 1892 by Isaac Roberts and later by the American astronomers (Keeler, Curtis and Ritchey), its peculiar filamentary structuro and afterwards to provide data for measuring its linear rate of expansion of about $0 \cdot 18^{\prime \prime}$ per annum.

Using this value and tho present angular dimensions of the nebula and extrapolating backwards, an interval of about 800 years is obtained for the nebula to expand from a point of origin. A similar time interval (900 years) was obtained by Hubble. Spectra of the Crab Nebula, first obtained in 1913-15 at the Lowell Observatory, showed a bowing of the emission lines, when the slit of the spectrograph crossed the whole extent of the nebula (major axis $6^{\prime}$ ). Interpreting this feature as a differential Doppler effect due to the approaching nearer side of a shell of gas and the receding further side, Mryall in 1937 derived a velocity of expansion of about $1,300 \mathrm{~km}$./sec. from Lick spectrograms. Assuming a constant rate of expansion, he concluded from the available data that the epoch of the outburst was about A.D. 1100. Meanwhile, Iundmark had pointed out that tho Crab Nebula was near the position of the bright object recorded in Chineso and Japaneso annals as having been seen for six months in A.D. 1054. In 1934, a translation by $Y$. Iba of the Japanese records gave the position of the object as near the star $\zeta$ Tauri and its brightness as equalling that of Jupiter. By combining the apparent linear expansion in seconds of arc per annum with the absolute ex. pansion in $\mathrm{km} . / \mathrm{sec}$, the order of distance was de. rived as 1,500 parsecs, equivalent to nearly 5,000 light years. Using this distance and the apparent magnitude of Jupiter $(-2 \cdot 2 \mathrm{~m}$.), the absoluto magnitudo of $-13 \cdot 1$ is obtained for the nova, which must have been at least one hundred times as bright as an ordinary nova.

In Contributions from the Mount Wilson Observatory No. 600, W. Baade assembles the evidence for the existence of two classes of nova, common novie and super-novx, which differ in luminosity by a factor of about 10,000 . Typical of the former class is the nova which appeared in tho Andromeda nebula in 1885 and reached a maximum apparent visual magnitude of $7 \cdot 2$, equivalent to an absolute magnitude of $-15 \cdot 0$. To this recently recognized class of super-nova, so the ovidence suggests, tho nova of 1054 may have belonged, and the expanding shell of gas originating with the cosmic explosion is still visible as the Crab Nebula.

\section{TREATMENT FOR ROT-PROOFING SANDBAG REVETMENTS}

\begin{abstract}
YANDBAG revetments exposed to the weather tend $D$ to break up, due to rotting of the sandbags, and inquiries have been made about preservatives. Two types of preservative are suitable. They are respectively a creosote or tar distillate, used as a water emulsion, or a solution of an organic copper salt in creosote made up into an emulsion. The former is more widely available than the latter and is suitable for treatment of revetments in position which have already deteriorated by being exposed to the weathor for some time. The latter is more potent. but is also more expensive, and its use will not generally bo justified unless the bags are in good condition and unless it is desirable to take down the whole revetment, treat all the bags and then re-pile them.
\end{abstract}

The application of the preservatives should conform with the following specification :

(1) A creosote or tar distillate of medium creosote type applied as a water emulsion in such a quantity as to give on the exposed portion of the bag a coating of creosote not less than one fifth of the normal dry weight of the fabric exposed. This is given approximately by. a 25 per cent creosote emulsion when sprayed on the bags to give a thorough coating. completely satisfying the absorption of the fibres, The creosote should comply with British Standard Specification No. 144/1936, "Croosote for preservation of timber'.

Any normal emulsifying agent may be used and the following is given as an example of the process of emulsification. 